\title{
OBSERVATIONS ON THREE HUNDRED CASES OF ACUTE MENINGITIS IN INFANTS AND YOUNG CHILDREN
}

\author{
L. EMMETT HOLT, M.D. \\ NEW YORK
}

With the introduction of lumbar puncture the diagnosis of acute meningitis entered on a new phase. This is quite as important an advance in this group of diseases as was the adoption of throat cultures in diphtheria and other throat affections. Exact diagnosis has now become possible where formerly in very many cases only a prubable diagnosis could be made. The period during which lumbar puncture has been systematically performed on all suspected cases in the Babies' Hospital covers the last five years.

The observations included in this paper relate to 300 cases of acute meningitis seen in this hospital, in children for the most part under three years of age. Of this number 197 have been observed during the lumbar puncture period and are available for more careful analysis. Some valuable data obtained from the records of 103 earlier cases are introduced. In order to gain some idea of the relative frequency of the different forms of acute meningitis I have not included in the table cases seen before lumbar punctures were regularly made nor cases of cerebrospinal meningitis observed during the epidemic which ended in the spring of 1906 , so that I think the figures which follow may be taken as fairly representing the usual conditions.

Etiologically, the 197 cases are divided as follows:

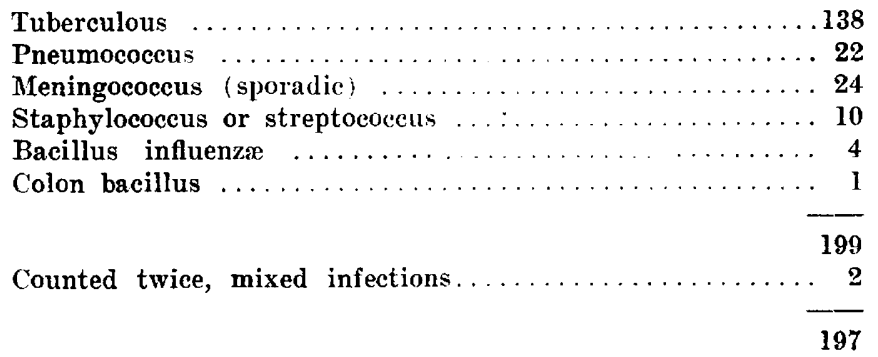

In other words, apart from epidemics of cerebrospinal meningitis, ro per cent. of the cases of acute meningitis occurring in young children are tuberculous. The objection may perhaps be raised that hospital figures are not to be taken as representing conditions which obtain generally. The admissions to the Babies' Hospital are, however, from every class, and I think this criticism will not hold. 
During the last five years lumbar puncture has been performed about one thousand times. After simple lumbar puncture no accidents have occurred; there have been no alarming symptoms, and no case of infection. However, in a single instance in which repeated injections were made in a case of cerebrospinal meningitis for the injection of Flexner's serum, secondary infection did occur, in spite of routine aseptic precautions. This is a warning which should be heeded. In another child suffering from cerebrospinal meningitis an alarming collapse followed the injection of 35 c.c. of serum. The fluid was warmed and the injection made in the usual manner, but the child came near dying and was rescued only after the most strenuous efforts. Aside from these two cases my experience has been without special incident. Too much, therefore, can hardly be said in favor of a systematic resort to lumbar puncture as a means of diagnosis in cases of meningitis; but it is a procedure requiring considerable skill and necessitating as strict asepsis as any surgical operation.

\section{SIMPLE MENINGITIS}

Before passing to a consideration of the different groups of cases which have been enumerated, I should like to say a word concerning "simple meningitis," a term formerly much used and one which still figures largely in vital statistics. In most such reports three forms of meningitis are given: tuberculous, cerebrospinal and simple. Some give only tuberculous as a separate class, combining cerebrospinal and simple. In the U. S. Census Report of the registration area for 1908 there are mentioned 71,229 deaths from meningitis in the five years 1904-1908; 25 per cent. of these are classed as cerebrospinal; 26 per cent. as tuberculous, and 49 per cent. as "simple or unqualified." In this same report, of 6,902 cases of meningitis occurring in the first three years of life in the year 1908, 32 per cent. are classed as tuberculous. In the New York City report for 1908 there are recorded in children during the first three years of life, 819 deaths from meningitis, divided as follows:

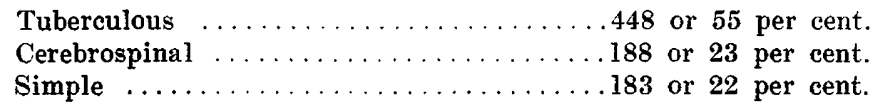

Previous to the period here considered the diagnosis of "simple meningitis" appears in the hospital records in twenty-three cases. It was at that time our custom to contrast such cases with those which were tuberculous. At present the group "non-tuberculous" meningitis has been pretty well differentiated. The term "simple meningitis" has no longer any significance and should be dropped. There is not the slightest doubt that the largest proportion of cases so classified belong in the tuberculous group. From experience in the hospital, where careful differential diagnosis of the different forms of meningitis has been possible by lumbar 
puncture, it is evident that tuberculous meningitis is very much more frequent than would appear from vital statistics of the country generally, or even those of New York City, and that a large number, probably the great majority of the cases put down as simple meningitis, and many of those recorded as cerebrospinal, are really tuberculous.

\section{COLON MENINGITIS}

Beginning with the smallest group I wish first to mention a case of meningitis due to a bacillus of the colon group which occurred in a baby only four weeks old. The child was admitted for an Erb's palsy and malnutrition. The usual symptoms of meningitis developed: temperature up to $103 \mathrm{~F}$., leukocytosis of 13,000, general hyperesthesia and rigidity. A purulent fluid was drawn by lumbar puncture which showed a pure growth of a bacillus of the colon group. The illness lasted ten days. There were no symptoms pointing to visceral lesions elsewhere, but no autopsy could be obtained. In private practice I have seen one other case of meningitis in which the only growth from the cerebrospinal fluid was a bacillus of the colon group. This also occurred in an infant four weeks old, who had previously suffered, it was reported, from a urinary infection due to the colon bacillus. The child recovered from the acute symptoms, but developed a secondary hydrocephalus.

\section{INFLUENZA MENINGITIS}

Thus far four cases of meningitis due to the Bacillus influenze have been observed; all of which have occurred during the last year. Clinically this form resembles the meningococcus cases and it is only by lumbar puncture that a differential diagnosis can be made. These cases are not common. I have seen but one other in private practice. Dr. Flexner tells me that he has had a considerable number of fluids scnt to him with clinical diagnosis of cerebrospinal meningitis which contained only the influenza organism. All these ended fatally, as well as those which have been personally seen. It is my belief that this is the almost invariable termination. In three of the four cases that have come under my observation the $B$. influenze was obtained by cultures from the bronchial secreticn during life, and in two, in which examinations were made, it was also obtained by culture from the nasopharynx. This would suggest the nasal mucosa as the port of entry. In three cases examined the organism was found in the blood. One recent case had some features of unusual interest. An infant of eight months was admitted to the hospital with an acute abscess of the left elbow. Cultures from the pus on ordinary media gave no growth; however, on blood-agar a pure growth of the $B$. infuenzoe was obtained. The child shortly after developed acute men- 
ingitis and died from the disease. The cerebrospinal fluid during life and the cultures from the meningeal exudate at autopsy showed a pure growth. of the $B$. influenza.

\section{MENINGITIS FROM PYOGENIC ORGANISMS}

The common pyogenic organisms, streptococcus and staphylococcus, have been very infrequently found by me as the cause of meningitis. They were present in but ten cases. In one other a streptococcus infection complicated a tuberculous case. It is rather striking that six of the ten cases occurred in newly-born children, and that in five the meningitis was secondary to spina bifida. In one it followed an operation on a hydrocephalus, and in only two cases did it occur under conditions resembling a primary inflammation. In one case, in an infant three months old, the autopsy showed encephalitis as well, and the streptococcus was also found in the heart's blood and lungs.

Clinically this form of meningitis was characterized by severe general and local symptoms and a rather rapid development, with always an unfavorable termination. Since so much has been made in medical literature of the association of otitis media and mastoiditis with acute meningitis, my experience on this point may possess some interest. I saw meningitis develop but once after otitis or its complications. This patient was a baby fifteen months old who had had a double mastoid operation done in Europe. Dressings had not been changed from the time the child had sailed until she was admitted to the hospital, a period of nearly two weeks. At that time they were very foul and the wound in bad condition, and the child desperately ill. Symptoms of acute meningitis developed and the child died in the course of a few days. The autopsy, however, showed the meningitis to be a tuberculous one. A careful examination showed no connection whatever between the mastoid lesion and the one in the meninges. My experience leads me to question whether the dangers of meningitis from cases of otitis or mastoiditis are as great as they have been represented. I have always in the hospital wards many cases of acute otitis and a moderate number of these patients develop mastoid complications. However, on by no means all of them is the mastoid operation done. I am, therefore, led to the opinion that so far as patients of this age, chiefly under two years, are concerned, meningitis from a neglect to perform the mastoid operation is one of the rarest occurrences.

\section{MENINGOCOCCUS MENIXGITIS}

It is not my purpose to enter minutely into a study of the twenty-four sporadic cases of cerebrospinal meningitis which have occurred since the great epidemic nearly five years ago. The number is too small to admit of any generalizations. Fourteen children received serum treatment, of 
whom six recovered and eight died. The deaths included one chronic case in which the patient was injected in the third month when in a hopeless condition. Another was one of the first patients to be treated by the serum; in this case only a single small dose was administered. One child was improving but died from intercurrent measles. Two children, one an infant of five weeks and another of nine months, died of marasmus after all symptoms of acute meningitis had subsided. One child at first improved very much under the injection of the serum, and all the organisms disappeared from the fluid, but the child steadily lost ground and died, the autopsy showing an encapsulated meningeal abscess at the convexity, containing fully half an ounce of pus which, by culture, showed the meningococcus. One child died suddenly a week after all acute symptoms had passed. No autopsy could be obtained. Death in one instance was due to secondary infection with the staphylococcus.

In several of our cases which ended in recovery the nervous symptoms were slow in disappearing. Inability to support the head sometimes existed for two or three months, and in two cases evidence of a moderate intraventricular effusion persisted for several weeks, but finally disappeared. I have been struck with the irregularity exhibited by these cases in infants, by the slowness of recovery and by the large doses of the serum required in those who did get well.

Of ten patients who received no serum, three recovered and six died; one was discharged unimproved and doubtless died soon after.

\section{PNEUMOCOCCUS MENINGITIS}

Twenty-two cases of pneumococcus meningitis were observed, or about 11 per cent. of the entire number. Of these, thirteen patients had definite pneumonia with consolidation and four of these had also empyema. One patient had pulmonary tuberculosis. Of the eight remaining patients near'y all had some pulmonary signs and symptoms, evidences of bronchitis at least. The diagnosis was confirmed by autopsy in eleven of the twenty-two cases. In twenty the pneumococcus was found in the cerebrospinal fluid; one of the two remaining cases coming to autopsy.

The histories of the two patients in whom lumbar puncture gave negative results have already been published ${ }^{1}$ but are sufficiently interesting to refer to again. While in only one of these was the diagnosis established by an autopsy, the symptoms in the second case were so similar that the diagnosis seems reasonably clear, though not positively proven.

The first was a child 20 months old whose attack came on with repeated convulsions, fever and a leukocytosis of 24,000. For one week the symptoms were those of a fairly typical acute cerebrospinal meningitis except for the absence of rigidity of the neck and extremities. Puncture made on the first day and repeated on the seventh day gave a perfectly

1. Arch. Pediat., March, 1908. 
clear fluid. No organisms of any kind could be discovered in the smears or by culture. During the third week the patient developed pneumonia with consolidation; and the persistence of the nervous symptoms led to a third puncture which, like the other two, gave negative results although the amount of fluid removed was large -105 c.c. After six weeks all the acute symptoms subsided and the condition resembled one of a mentally defective child with slow wasting. Death occurred in the eleventh week from a second attack of pneumonia. The autopsy showed an old meningitis at the convexity; from this exudate a pure growth of the pneumococcus was obtained. The base of the brain was normal.

The second case occurring in a child 15 months old was almost the counterpart of this first one, but ended in partial recovery. Three punctures were made, in all of which the fluid was negative. Pneumonia developed in the fourth week but cleared up. Active nervous symptoms continued for four weeks, then gradually passed away. When discharged from the hospital the patient could not sit up nor support the head. Vision and hearing seemed absent. Six months later the patient was seen again. There had been considerable improvement, but the child clearly was mentally defective.

It seems evident that there was in both of these cases some involvement of the cortex in the inflammatory process; but no paralysis occurred in either case. Neither in its symptoms nor in its course did the disease suggest the cerebral form of poliomyelitis. We are accustomed to place so much reliance on lumbar puncture that the possibility of the existence of acute meningitis with a normal fluid is rather difficult to grasp.

Clinically, pneumococcus meningitis is shorter than any of the other forms, possibly excepting that due to influenza. In eight of the twentytwo cases it lasted three days or less; and in two-thirds of the entire number it was six days or less. It presents greater difficulties of diagnosis than any of the other forms; in the first place for anatomic reasons. The lesion is usually most marked at the convexity and may be limited to that part of the brain. The diagnosis of a convexity meningitis is proverbially difficult, for many of the most characteristic signs such as opisthotonos, irregularity of pulse and respiration, cranial-nerve involvement and distention of the fontanel, are usually wanting. Again, pneumococcus meningitis has been in my experience seen in younger patients than either cerebrospinal or the tuberculous form. Half of our cases occurred in infants under six months old, and two-thirds were under nine months. It has long been my belief that pneumococcus meningitis, like pneumococcus pericarditis and peritonitis, was the result of a generalized pneumococcus infection occurring much more frequently in very young patients than in those who were older. This has been confirmed by finding the pneumococcus in the heart's blood in every one of seven cases of pneumococcus meningitis in which cultures have been made. 


\section{TUBERCULOUS MENINGITIS}

I have already said that 70 per cent. of my cases of acute meningitis, apart from the epidemic of cerebrospinal meningitis, were tuberculous. Its great frequency I am sure is not appreciated. During the past four years 8 per cent. of the deaths in the Babies' Hospital have been from this cause. I may have overlooked some cases but I think not many; for autopsies are obtained in this institution in nearly 50 per cent. of the fatal cases, which is a larger proportion than in any other hospital of the City so far as my knowledge goes. Besides, every suspected case receives lumbar puncture and the cerebrospinal fluid is examined both microscopically and by culture. We may safely say that none have been wrongly diagnosticated, for in all but two cases bacilli have been found.

In general practice tuberculous meningitis is certainly very often overlooked or a mistaken diagnosis made. Of thirty successive cases admitted to the hospital, in only three was the diagnosis of tuberculous meningitis previously made and in only three others was it suspected. 'The most common diagnosis with which the child was admitted was either some gastric disorder or cerebrospinal meningitis.

There are two common misconceptions regarding tuberculous meningitis: the first is that it is a disease of long duration, olten lasting two or three months or even longer. I have never seen tuberculous meningitis last over five weeks, and only once have I seen it last five weeks, this in a child of nine years. Of the cases reported in this paper, in infants chiefly, the average duration has been about two and a half weeks after the first definite symptoms of illness appeared. A second error is that tuberculous meningitis usually affects delicate infants, those suffering from malnutrition, often of the marasmus type. The inference is drawn that because a child with symptoms pointing to meningitis was previously in good health, the process could not be tuberculous. It is my experience both in hospital and private practice that the vast majority of these patients have been, previous to their attack, apparently in as good health as any children whom I see. Not that very healthy infants are more prone to tuberculous meningitis than others, but that a tuberculous infection of a young child is very apt to involve the brain early, before there is time for the symptoms which result from a general tuberculosis to be manifest. Some reasons for this will appear later.

Seasonal Prevalence.-That tuberculous meningitis occurs more at one season of the year than at another has not, so far as I am aware, hitherto been noted. For the last two or three years I have been struck with the fact that more patients were admitted during the spring months than at other times and I have had the earlier records of the hospital searched to ascertain the month when patients were admitted. 
Chart 1 shows by months the time of admission of 218 patients in the Babies' Hospital service. It will be noted that the curve is at its lowest during the summer and early fall; that there is a slight rise in December and January, but a very sharp rise in the spring months, the highest point being touched in the month of April; the number of cases in the spring months of March, April and May being about twice as great as were seen at any other similar period during the year. A steady fall is noted during the summer until October is reached.

The explanation for this variation is not altogether clear. The most plausible one seems to be that these infants have been infected some time previously and are carrying a latent focus somewhere in the respiratory tract, usually the bronchial glands. Under the influence of acute respira-

CASES OF TUBERCULAR MENINGITIS

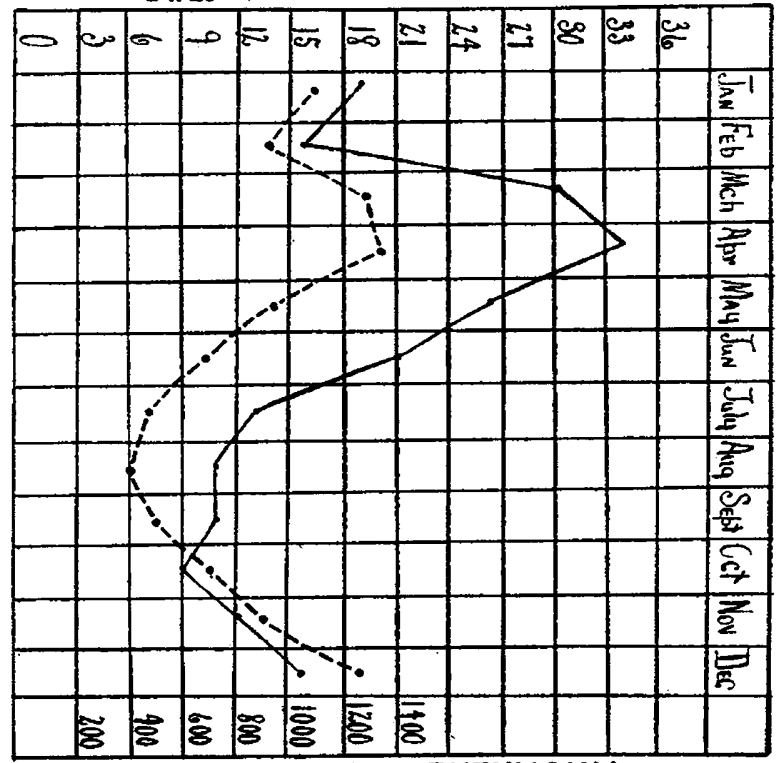

DEATHS FROM PNEUMONIA

Chart 1. The upper curve represents the seasonal occurrence of 218 cases of tuberculous meningitis; the lower curve represents the deaths from pneumonia in New York City for one year.

tory infections occurring during the winter and spring months, the latent tuberculous disease becomes an active process and a rapidly spreading infection occurs; for in none of these infants is the brain lesion the only one. At autopsy pulmonary lesions are almost invariably found; and miliary tubercles are usually present in many other organs. This indicates that the meningitis in infants is a part of a generalized infection and causes death before the other evidences of the disease are usually manifest. Some of these children showed a few scattered râles in the chest; 
and, still more infrequently, evidences of more serious pulmonary disease were present; but the great majority of them showed no pulmonary signs of disease whatever.

An attempt has been made to see in how many cases of tuberculous meningitis bacilli could be found in the sputum. In many it has been difficult to secure any proper sputum for examination. Yet in 116 patients in whom the effort was made, bacilli were found in forty-nine. These cases were taken routine, not selected because of the presence of pulmonary signs or symptoms. It is true that the number of bacilli is seldom large and unless careful and sometimes repeated examinations are made, they are likely to be missed. Yet when present they were found

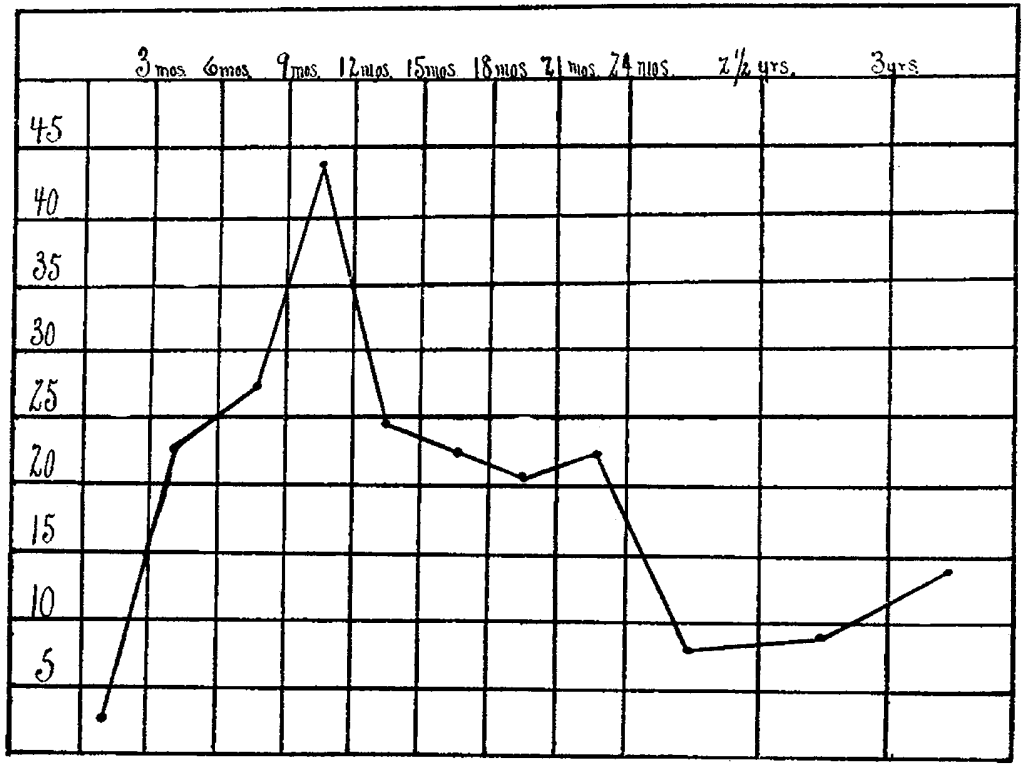

Chart 2. The age incidence in 218 cases of tuberculous meningitis.

at the first examination in three-fourths of the cases, although in one case, not until the sixth examination.

The close association of tuberculous meningitis with inflammations of the respiratory tract is also suggested in the accompanying chart. The lower curve in Chart 1 shows the deaths from pneumonia in New York City for 1909. The similarity of these two curves is certainly striking. I do not think that the tuberculous meningitis in older children would show so close an association with pulmonary disease as is true in infancy.

Age Incidence.-Chart 2 shows the age incidence. Inasmuch as the Babies' Hospital admits children up to three years of age the curve I think may be taken to represent pretty accurately the occurrence of the disease in patients below that age. Only three patients under three 
months of age were seen; the greatest number of patients were seen between the ages of nine and twelve months, a rapid decline in the curve being noted after the first year. This is a striking fact, but the reason for it is not very clear. The greater frequency of tuberculous meningitis in children from nine to twelve months of age may be due to the closer exposure of young infants to infected adults than occurs with children who are somewhat older. It is possible also that a closer exposure occurs in winter than in summer and that this may have some bearing on the greater frequency of the disease in the spring months. The incubation period is at present unknown. It seems probable that in most cases it is several weeks at least.

Source and Type of Infection.-It is my experience that nearly all the cases of tuberculous meningitis, the source of whose infection we have been able to trace, have their origin in exposure to adults with pulmonary tuberculosis. A striking confirmation of this is shown in the results of the observations made by Dr. W. H. Park in the Research Laboratory of the Health Department on thirty-two cases in my series in which the type of bacillus was worked out. In thirty it was of the human type; in one it was of the bovine type; in one case both types were present.

The von Pirquet Reaction.-The statement frequently has been made in medical literature that comparatively few infants with tuberculous meningitis respond positively to the von Pirquet test. Systematic observations on this point have been made during the past two years. Of sixty-five cases a positive reaction was obtained in fifty-one; a negative in eleven, and doubtful in three. Of the fourteen cases that were negative or classed as doubtful, four patients were moribund and seven extremely prostrated and with feeble circulation. In the remaining three there was no apparent reason why a reaction should not have been obtained. I regard the skin test, therefore, as of very great diagnostic value, and a negative result, except in the conditions just mentioned, of much diagnostic importance.

I have attached so much importance in recent times to the von Pirquet test and the findings of the examination of the cerebrospinal fluid, that negative results in either of these I have come to regard as very significant. A baby seven months old was admitted with a fairly typical history of tuberculous meningitis: onset with vomiting, slight fever, convulsions developing in three or four days; drowsiness merging into stupor; marked constipation. The prominence of the fontanel was so great that the mother herself had noticed it and, in fact, the "lump on the head" was one of the reasons why the child was brought to the hospital. Lumbar puncture was made and 120 c.c. of perfectly clear fluid removed. It was under very high tension. So confident was $I$ that this was a case of tuberculous meningitis that the search for the bacilli in the cerebropsinal 
fluid was continued for several hours, but with negative results. Greatly to my surprise the child did not respond to the von Pirquet test, but what was still more unusual, the child promptly recovered and at the end of a week was discharged to all appearance quite well. It died about one month afterward rather unexpectedly, but without cerebral symptoms, so the visiting nurse learned. I have seen an almost identical picture in another child, in whom recovery was complete and permanent. The explanation of these is rather difficult without assuming the existence of a primary acute serous meningitis, regarding which $I$ have always been rather skeptical.

\section{CONCLUSIONS}

Lumbar puncture has placed the diagnosis of meningitis on a new basis. It should be employed in every suspected case. It is only with very rare exceptions that to a skilled observer it gives uncertain results.

Nearly three-fourths of the cases of acute meningitis in young children are tuberculous, except when cerebrospinal meningitis is epidemic.

In tuberculous meningitis bacilli are always present in the cerebrospinal fluid; although difficult to find in the early stages, in the later stages a careful examination should discover them. The von Pirquet test in most cases gives positive results.

In infants and very young children tuberculous meningitis occurs usually as an early manifestation of a general tuberculosis, and generally causes death before the lesions elsewhere are far enough advanced to give definite signs or symptoms. Pulmonary lesions are present in nearly all cases.

Tuberculous meningitis is of human origin. Altogether the most frequent cause is exposure to adults with pulmonary tuberculosis.

The age incidence and the seasonal occurrence are points which are not yet fully explained.

Pneumococcus meningitis usually occurs in younger patients and is associated with pneumonia. Clinically it resembles meningococcus meningitis but usually the course is shorter. It is almost invariably fatal.

Influenza meningitis is infrequent and also resembles the cerebrospinal form. It is almost invariably fatal.

Streptococcus and staphylococcus meningitis are very rare forms in jnfancy, usually occur in the new-born and are often associated with spina bifida. Under such circumstances they are always fatal. From mastoid disease this form is seldom seen in young children.

Meningococcus meningitis is really the only variety in which at present there may be said to be any hope of recovery. The results in this variety of meningitis have been very greatly improved by the serum treatment.

14 West Fifty-Fifth Street. 